\title{
RETHINKING LANGUAGE AMBIGUITY BEYOND THE SEMANTICO-PRAGMATIC INTERFACE
}

\author{
Khadija Belfarhi, University of Badji-Mokhtar Annaba, Algeria, \\ khadija_belfarhi@yahoo.com
}

Original scientific paper

DOI: 10.31902/fll.34.2021.12

UDC: 81'27

\begin{abstract}
Ambiguity occurs when the language user intends unspecific and unclear meaning through written or spoken language. It has been approached by semantics, pragmatics and philosophy of language because indeterminate meaning needs a mechanism that assembles the right attributes for decreasing ambiguity. In that, such a mechanism acts away from the structural conditional which was for long thought applicable for clarifying meaning. An extension in the semantic-pragmatic mechanism is needed as far as the latter fails to account for indeterminate meaning. The present paper suggests a more interactional mechanism that integrates two components. The former relies on the linguistic resources that act as possible attributes including both the semantic and pragmatic. The second, however, is the free component wherein the analyst acts in free path that does not necessarily follow the linguistic rules and the fairplay aims at extending the attributes and their interaction. The application of this mechanism has been on a variety of ambiguities ranging from the simplest daily metaphors to the most indeterminate utterances. It has been found that its application succeeds with distanced attributes, as when the utterance's meaning is carried out by another far attribute.
\end{abstract}

Keywords: ambiguity, linguistic analysis, extension, interactional, free component, pragmatics, semantics, approximation, substitution, non-realization

\section{Introduction}

The linguistic mechanism is capable of accounting for linguistic meaning, i.e. the type of meaning which makes sense to both the sender and the receiver. Speech vagueness may hide in the intentions unrevealed by the speaker. When the addressee fails to decode the message, meaning is thus intentionally ambiguous. Ambiguous meaning has been for long the task of linguistic semantics standing on the principle of compositionality which looks at meaning as complex expression gaining meaning from a function of the meanings of its constituents and the way they are combined. But compositionality focuses on structural conditioning and makes ambiguous meaning out of reach and needs another mechanism. 
An alternative view is that meaning which resides within the pragmatic paradigm has more extensional scopes through the cooperative principle which looks at meaning from the truth conditional scope while the intended meaning needs rather, as Grice put it, an actional perspective to reach the speaker's intent through inferences. Pragmatically speaking, this is m-meaning or "meaning intention." (Grice 1957, 1968). However, the cooperative principle is questioned for its ability to account for ambiguous meaning as the latter is inherently vague and indeterminate. And "many instances of intentional behavior may better be characterized as emergent products of complex selforganization processes" (Gibbs-19).

The linguistic analysis with its semantico-pragmatic extensions is still incapable of explaining ambiguity in both the spoken and written language. Such a meaning needs a more functional mechanism which adapts to the variability of the input and can approach ambiguity by integrating a free component capable to cope with the semantic indecisions for its openness on a logical reasoning contributing in understanding ambiguity.

\section{Ambiguity in Language}

Ambiguity in language was for long attributed to lexical ambiguity, though non-lexical sources present other varieties. Lexically speaking, "Ambiguity describes the linguistic phenomenon whereby expressions are potentially understood in two or more ways; an ambiguous expression has more than one interpretation in its context" (Wales -387). Sentence ambiguity can be lexical when a single word has more than one meaning, or when a word can be read in more than one way (Dayal 2004). Structural ambiguity occurs when a sentence has more than one deep structure. It is the case of semantic ambiguity with a sentence having more than one way of reading within its context although it contains no lexical or structural ambiguity (Cruse 1986). A word, expression, sentence or utterance may occur with one potential meaning when the speaker is explicit in his use of language. It may, however, have more than one meaning when the speaker intends to make his/her use of language unclear and, therefore, ambiguous. Cruse talked about establishment of senses which is presumably represented differently in the mind's lexicon (68). The established senses, or at least one of, do not make relevance to the language receiver, and may, consequently result in semantic ambiguity.

Ambiguity in language has been the concern of semantics, semiotics and the philosophy of language as meaning may occur out of the denotative scope especially when the speaker intends to make his 
utterances/sentences ambiguous. Carston considers language ambiguity as part of language use and not the semantics of the utterance/sentence:

It's not a semantic ambiguity because it doesn't reside in lexical or syntactic ambiguity, that is, in the linguistic system itself. It is a matter of speaker use of the description, specifically of the sort of intention that informs the use. This is a particularly significant turn of thinking, since while the referential/attributive distinction is conceived of as a pragmatic matter, it appears also to be a truthconditional ambiguity, in that different propositions are expressed on the two uses (8).

The indeterminacy of signs has been studied, too, by philosophical logic whereby earlier logicians had questioned the unclear relation between the signifier and its attribute. Ambiguous meaning cannot be found in the concept itself as argues "the thing itself" -philosophy. Approximation is rather more plausible for non-sensitive meaning because to find the identical meaning of a given word or utterance is not within the simple equation " $\mathrm{x}_{1}=\mathrm{x}_{2}$ ".

If $x_{1}$ is a signifier shaped linguistically

And if $x_{2}$ is its attribute but varies and lacks a linguistic shape;

Therefore, $\mathrm{x}_{1}$ is indeterminate.

In linguistic meaning, $x_{2}$ is understood either in itself or approached by means of implications along the speaker-hearer cooperation. In ambiguous meaning, $x_{2}$ situates somewhere in a nonsensitive form in the context of production. To find its meaning is principally a task that no longer looks at the target utterance or its attribute. The utterance's meaning situates then in an instance which can be near to the utterance or far from it; and carried out linguistically or metaphysically. In both cases, meaning's first face is linguistic a reason why linguistics, or, more particularly, semantics takes a major role in the search of meaning. Linguistic semantics looks at meaning from the associative principle stating that the meaning of $\mathrm{x}$ is carried out by the meaning of its associates. In this way, meaning assigns to the following dogmas summarized by Szabó:

The first dogma: an expression is meaningful if and only if there is a semantic value $m$ and there is a relation $R$ such that the expression bears $\mathrm{R}$ to $\mathrm{m}$.

The second dogma: provided that an expression is meaningful and given the way the world is its meaning determines its referent. 
The third dogma: The principle of compositionality e1, e2, ... are $\sigma(e 1), \sigma(e 2), \ldots, \sigma(e n)$, then the semantic value of fis $\sigma(\sigma(e 1), \sigma(e 2)$, ..., $\sigma($ en $))(32-52)$.

The principle of structural conditional applies to all those types of meaning where the referent is carried out by a referent that either manifests in the referent or its meaning determines its referent according to its occurrence in a given context. The referent is for long thought to be the task of the semantic analysis as the latter's role is to provide interpretation to any component generated by the syntactic component. The semantic theory draws heavily from the compositional principle in the interpretation of utterances: "The process by which a speaker interprets each of the infinitely compound constituent of a sentence is obtained as a function of the meanings of the parts of the constituent" (Katz-297). The compositional meaning requires syntactico-semantic knowledge to determine meaning from the combination of its constituents. This is a linguistic projection of meaning relying on the way syntactic constituents are composed. According to Katz, the semantic component comprises a general component which is the word's meaning as put in the dictionary, and a projection component which derives meaning on the basis of semantic markers. He says:

A system of projection rules that provide the combinatory machinery for projecting the semantic representation for all supraword constituent in a sentence from the representations that are given in the dictionary for the meanings of the words in the sentences. (298)

If the principle of compositionality is to look at from the side of the composed elements themselves and, say, if $\sigma\left(e_{1}\right), \sigma\left(e_{2}\right), \ldots, \sigma\left(e_{n}\right)$ combine together, do they lead to $\sigma$ ? The answer is yes if it is to rely on the semantic-syntactic knowledge. For example, the following order "Marry", "went" and "early" should lead necessarily to the meaning of the performed action as it is $R$ to $M$ without calling for an implied meaning. But are the semantico-syntatic resources always sufficient to lead to compositional meaning? The answer depends on whether the aim of the utterance is to be understood or not. If the utterance does not make sense to the language speaker and it is judged to be indeterminacy, then the right side of the equation fails to equalize the left side.

The relation is tripartite between compositionality, sentence's meaning and speaker's meaning. An utterance or a sentence has a 
meaning as the result of a structural agreement between its components with the condition that the speaker aims at explicit meaning. When the speaker does not aim at explicit meaning and makes his utterance/sentence ambiguous, the structural agreement holds but does not say a lot on what the speaker intends to mean, and from here arises the ambiguity of the utterance/sentence. In other words, compositionality is not a necessary condition for explicit meaning and another order of utterance may require navigation along what the speaker intends to mean rather than what his utterance/sentence means in relation to its structural state.

\section{The Semantic Ground}

What can decrease ambiguity is the grounding which is likely to yield m-intention. The latter is a built meaning from the speakers' grounding which contributes to meaning making as it is established in the outset and which is subject to gradual change over time by means of socioculturally newly attributed relations. For example, the following two daily expressions are used in English but they have developed differently:

(1) Go with the flow

(2) Zombie bank

The former has origin in Shakespeare's Julius Caesar and it is frequent in spoken English while the second one is newly attributed expression dating to the 1999s in relation to financial crisis. The former kept a stable meaning since Shakespeare while the second makes sense in the last decades as it attributed a new meaning. Had this expression been uttered in, say, the $18^{\text {th }}$ century, it wouldn't have made sense simply because speakers at that time lacked the current ground of insolvent bank that survives through government support. The ground is, therefore, built by the newly attributed meaning added to the linguistic ability of speakers as it develops conventionally, and any decrease in the ground vis-à-vis the participants may make it less contributing to m-meaning, or, in other words, it has minor contribution.

The grounding can be purely cultural as speakers develop special ways of saying the same thing through time, and which can be seen as culturally sensitive meaning. For example, consider the following sentence uttered by a leader of a political party in Algeria as a comment on the Arabic spring:

(3) Like beans like lens

If the sentence was said to a non-Algerian speaker, even an Arab person from another country, it would make no sense and it would remain as an intentional ambiguity because it is about two different 
vegetables. It is locally understood as two similar situations, through which the addresser means that before and after the Arabic spring, no great evolution can come. In that, before the Arabic spring people eat beans and after the Arabic spring they eat lens. In fact, ambiguous meaning is a rhetorical device that competent speakers employ to avoid long clarifications as just one expression fits.

In the previous example the m-intention is not impossible to reach as an attentive addressee can come to the speaker's intent. In the same field of politics, it happens to hear very ambiguous meaning which resists interpretation because the grounding is neither developmental nor cultural. President George W. Bush, for instance, was known for his unclear meaning ${ }^{1}$ :

(4) The vast majority of our imports come from outside the country.

This sentence has been considered as a memorable misstatement, and English native speakers would think about it as nonnormative or simply nonsensical utterance. However, this is the general view of the general addressee of the utterance. Critical discourse analysis considered the utterances differently. Silverstone (2003) sees it on message: "Single terms can become 'a shortcut for understanding the world', because they are 'particularly precise in suggestively communicating the identity "who" and the contextual "why" of their use" (Blommaert-135). Section 4.1 below will take up the example (4) for further analysis.

\section{Semantics vs. Pragmatics}

Any sentence is subject to the distinction between the linguistic meaning and the propositional meaning or, if better, sentence meaning and utterance meaning. The former is stable in the sense that it applies to linguistic rules which are context-independent while the former is context-sensitive and depends strongly on the context whereby the more the context is unclear the more the utterance's ambiguity increases. Recanati (2004) draws a tripartite distinction between the sentence meaning, what is meant and what is implicated. In that, the first is context-independent whereas the second and the third are context-sensitive. However, the difference between the two is that what is meant cannot go beyond the semantic potential of the sentence and the worked propositions keep semantically driven. The second, what is implicated, navigates along the long chain of inferences. Recanati (2004) argues that the interpretation of the sentence meaning does not

\footnotetext{
${ }^{1}$ Wilson, 2015.
} 
necessitate more than a minimalist interpretation while the implicated meaning requires a non-minimalist work open to more advanced inferences lying beyond the primary linguistic meaning.

The semantic interpretation is a minimalist account for sentence meaning working at specific level of words' meaning which keeps within the semantic potential of the utterance. The pragmatic interaction is rather out of the sentence, operating in a large context. Recanati distinguishes semantics from pragmatics as follows:

Semantic interpretation is the process whereby an interpreter exploits his or her knowledge of a language, say $L$, to assign to an arbitrary sentence of $\mathrm{L}$ its truth-conditions. Pragmatic interpretation is a totally different process. It is not concerned with language per se, but with human action. When someone acts, there is a reason why he does what he does. To provide an interpretation for the action is to find that reason, that is, to ascribe the agent a particular intention in terms of which we can make sense of the action (54).

Despite the fact that both semantics and pragmatics are theories of signs, they approach language ambiguity differently and disagree about the role of terms such as intention, implication and context, grouped as the pragmatic conditioners. The problem with semantics is its limited focus on sentence's meaning by virtue of the truth conditional content while pragmatics' problem is the limited focus on context. According to Recanati, delimiting the context is problematic as there is no limit to the amount of contextual information, making the pragmatic interpretation subject to defeasibility.

Another position along the distinction between semantics and pragmatics sees the latter as generalization of semantic notions. In his discussion of the distinction between semantics and pragmatics, Szabó states different views among them Montague's characterization which takes pragmatics as extension of the semantic techniques claiming that "pragmatics does not abstract away from designata, and so it becomes an extension of semantics, not a distinct field" (8). Montague aimed at specifying all the features of the context of utterance that play a role in determining extensions. However, this is not feasible, as noted Szabó as "[t]he chances of listing all the features of the context upon which extensions in natural languages depend seem bleak" (9). The context can, as Szabó noted "include at least some information about speaker intentions" (34). Szabóimplies both the semantic and pragmatic 
components in utterance interpretation, as one cannot operate without the other:

We understand semantics and pragmatics as subfields within the general study of utterance interpretation, the process whereby the addressee determines what the speaker meant in uttering a linguistic expression. Typically, but not always, such a process will include a component when the speaker associates linguistic expressions with their meanings: this is the subject-matter of semantics (23).

The truth-conditional content may not be relevant to the interpretation of ambiguity because the same sentence may have different truth conditions. Carston (2) says:

The concept of 'semantics' at issue in the semantics/pragmatics distinction as construed here, not to be equated with truth conditions. According to this picture, a truth-conditional semantics cannot be given directly to natural language sentences but should take fully propositional thoughts as its proper domain.

The ambiguous utterance is primarily a sort of communicated thought and not a strictly linguistic utterance: "thoughts, whether expressed by utterances or not, are semantically complete, that is, that they are truth-evaluable in and of themselves, without any need of contextual completion or specification." (Carston-31).

The real world is represented by language and the speaker's perception of it; that is, the encoding of objects in the real world is realized at the syntactic level via language and at the deep level, it holds other conditioning elements. Carston drew a distinction between semantics and pragmatics standing on the relevance theory. It is a distinction between the two types of cognitive process employed in understanding utterances: decoding and inference (Carston-2). In other words, the semantics/pragmatics interface is the point of contact of the linguistic parser and the inferential mechanism(s)(ibid-7). Carston brings both semantics and pragmatics in one cognitive line:

The linguistically encoded element of an utterance should not generally be geared towards achieving as high a degree of explicitness as possible, but should rather take account of the addressee's immediately accessible assumptions and the inferences he can readily draw (15). 


\section{Free Reasoning: A Philosophical Perspective}

The semantic-pragmatic distinction discussed the roles of both the semantic value of the utterance, and the contextual information in different orientations which are dictated from the underdeterminacy of utterances leading to inability to settle on one particular formal approach. The latter needs more than the semantics of the utterance, and needs, too, more than the contextual information. It needs a nonformal approach implying a rather philosophical endeavourer that copes with the variability of ambiguous utterances.

The difficulty in yielding meaning of ambiguous utterances lies principally in the inability of the linguistic analysis to account for a variable meaning with the application of a fixed set of semanticosyntactic processes. Ambiguous meaning, in its construction, is put purposively vague and, therefore, complex. Its interpretation should then look for a mechanism that covers the variability of signs if the latter occur in a closed context. In that, pragmatic cooperation fails to clarify ambiguity because it is not an automated process in the sense that it can resolve all ambiguities. Wilson and Sperber note in this respect:

Most would also agree that pragmatic interpretation is ultimately a non-demonstrative inference process which takes place at a risk: there is no guarantee that meaning constructed, even by a hearer correctly following the best possible procedure, is the one the speaker intended to convey (4).

Two levels can be distinguished as far as understanding ambiguous meaning is concerned. The first is the one that despite the ambiguity of the utterance, "only a hearer with some advanced knowledge of at least the gist of what the speaker might have wanted to convey would find it relatively easy to reconstruct the intentions behind her utterance using a rationalization procedure" (Wilson and Sperber 10-11). The second, zero meaning from the surface level ${ }^{2}$, is the one that fails to be read or understood as "the standard procedures for inferring intentions do not help with identifying the speaker's meaning" (ibid, 11). In fact, a third category of ambiguous meaning is the written word in a coded medium. In the absence of the Jacobean communication, and when the Gricean cooperation becomes addressee-word instead of addresser-wordaddressee, meaning put intentionally ambiguous breaks the standard

\footnotetext{
${ }^{2}$ This is the case of those utterances that have their words put in an order not clearly seen in the text. Toolan (10) calls it a 'zero prospection' or not-a-text describing les communicative literary texts quite similar, Derrida calls such utterances as "sleeping" or in French "sense dormant".
} 
ways of understanding and interpretation. Ambiguity at the spoken level is less problematic than at the written ${ }^{3}$ as instantaneity allows participants to get to meaning if not by the token utterance it can be by other contributors of the pragmatic cooperation. At the written level, thus, ambiguity is critical as the cooperative elements are all coined in the written word. Examples of this can be single meaningless words or expressions, ambiguous implications and presuppositions, ellipses, metaphors, full grammatical expressions or sentences with zero meaning in the text, disjoint words and expressions.

An alternative trajectory on understanding ambiguous meaning claims throughout this paper that a linguistic utterance performed ambiguously and along non-objective way needs not always an objective mechanism of the sense speaker-utterance-hearer. The cooperative mechanism cannot resist to the ambiguity of meaning as the latter is produced by an intent different from that of the conventional mechanism. Recananti (56-57) says in this respect:

Any piece of contextual information may be relevant. But the context that comes into play in the semantic interpretation of indexicals is not the total pragmatic context; it is a very limited context which contains only a few aspects of the pragmatic context [...]. But context-sensitive expressions are semantically underdeterminate rather than indexical in the strict sense. A possessive phrase such as 'John's car' means something like the car that bears relation $R$ to John, where ' $R$ ' is a free variable. The free variable must be contextually assigned a particular value; but that is not determined by a rule and it is not a function of a particular aspect of the narrow context but as a function of what the speaker means.

Free reasoning implies adopting a dynamic way of approaching ambiguity capable of surrounding the ambiguity from different angles if they are attributes of meaning. The angles can be semantic and pragmatic as both supply the analysis with the basic attributes that act as a platform for carrying up the analysis. A free path of a pure philosophical nature is added to the semantico-pragmatic component to enable the analyst to move freely along those attributes that carry

\footnotetext{
${ }^{3}$ Meaning is much indeterminate in the written medium because in the spoken medium, speakers take into consideration the perspective of the listener to make intentions clear. Besides, the spoken medium has utterances performed within the participants' common ground. In that, speakers are generally guided by the we-intention (Searle 1990).
} 
meaning. A raised question here is why to go along free reasoning while semantics and pragmatics are supposed to clarify ambiguity. The issue lies mainly in word-world relations shaped by language users. The relation between the word and its meaning is not always unidirectional. Ambiguity is abstract and vague and does not obey to the compositionality principle. Pragmatics' contribution lies in the openness to contexts, implications and presuppositions supposed to clarify meaning. Pragmatics can contribute to the clarification of ambiguity since its task is the study of the mobilization and use of the linguistic as well as non-linguistic resources in the production, transmission and interpretation of meaning. In that, semantics deals with utterances' meaning and pragmatics is concerned with the speakers' meaning. Pragmatics is the study of certain regularities in the causation of utterances underlying the practical functions they serve (Stamp 1981). Pragmaticsis operational within a regular pattern and cannot navigate along the irregular. The latter refers to irregularity in relations between words and their referents. The free reasoning, however, is open and free from any restriction and does not obey to a conventional mechanism. Words may contain multiple senses as part of their semantic structure. Meaning is cast upon its real use which assigns sets of readings. The free reasoning allows for a delimitation of readings on the basis of substitution of attributes and approximations in a wide context.

\section{Substitution of Attributes}

When thinking about how to understand ambiguous meaning, one confronts at first the notion of multiplicity of utterances at the paradigmatic level. That is, a word, spoken or written, can manifest by its linguistic meaning, which is the common one to understand with the basic linguistic analysis, and can, too, manifest by another meaning which is distant from it along different degrees as the more distant the more meaning gets far from the addressee's reach and vice versa. This multiplicity corresponds, in fact, to the levels of inferences the addressee goes through: "several levels of metarepresentation involved in inferential comprehension" (Sperber and Wilson 2012). If meaning is constructed in a scheme incompatible with the Gricean, its understanding, then should cope with a receptive mechanism which should be decoded in a different scheme. In other words, if the production of meaning adopts non-objective scheme, its understanding, too, should adopt a non-objective scheme featured with vertical reading along open paradigms because ambiguous meaning is intentional, as said Van Orden and Holden (2002) "intentional actions self-organize as embodied, vertically coupled, control processes" (95). 
Verticality of values originates in the paradigmatic relation between words. Words have primary and secondary values in the sense that these values may interact with each other when the addressee, whether at the spoken or written medium, aims purposively to distance the meaning of his utterance or word from the source meaning. A simple example starts from an ordinary metaphor:

(5) Broken hearth

If we analyze the two words into their primary and secondary values, we find the following ${ }^{4}$ :

Broken: Divide into pieces

To snap off or detach

To crack without separating into pieces

To vary or disrupt the uniformity or continuity of

Heart: A hollow muscular organ that pumps the blood through the circulatory system by rhythmic contraction and dilation.

The vital center and source of one's being, emotions, and sensibilit ies.

The repository of one's deepest and sincerest feelings and beliefs Emotional constitution, basic disposition, or character

Capacity for sympathy or generosity; compassion

Love; affection

The metaphor's meaning lies in the interaction between one value of the verb "break" and another value for the noun "heart". The interaction between "break" and "heart" is in terms of the primary value of "heart" and the secondary value of "break". But this is not all: there is a modulation in the line between as the comparison acquires along a new attribute that does not apparently exist in both words but brings them together in one meaning which is feeling hurt and sad:

Break

Divide into pieces

To snap off or detach

To crack without separating into pieces

To vary or disrupt the uniformity or continuity of

4 The definitions are taken from the free dictionary at www.thefreedictionary.com 


\section{Heart}

A hollow muscular organ that pumps the blood through the circulatory system

by rhythmic contraction and dilation.

The vital center and source of one's being, emotions, and sensibilities.

The repository of one's deepest and sincerest feelings and beliefs

Emotional constitution, basic disposition, or character

Capacity for sympathy or generosity; compassion

Love; affection

\section{Divide into pieces}

\section{Love; affection}

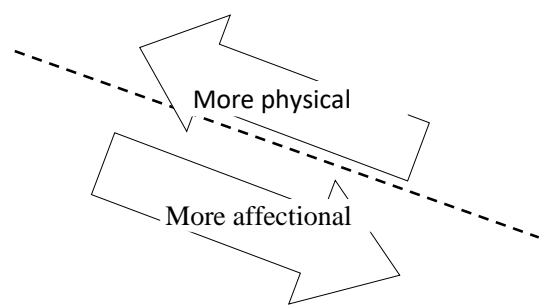

Both words, thus, interact at the level of affection resulting in the deep value "hurting feelings". Both words, therefore, have their values interacting at one level which becomes then the meaning of the expression. A similar interaction but in an opposed side occurs with the Algerian metaphor "heart iron", and also the English metaphor "iron heart":

\section{Heart}

Iron
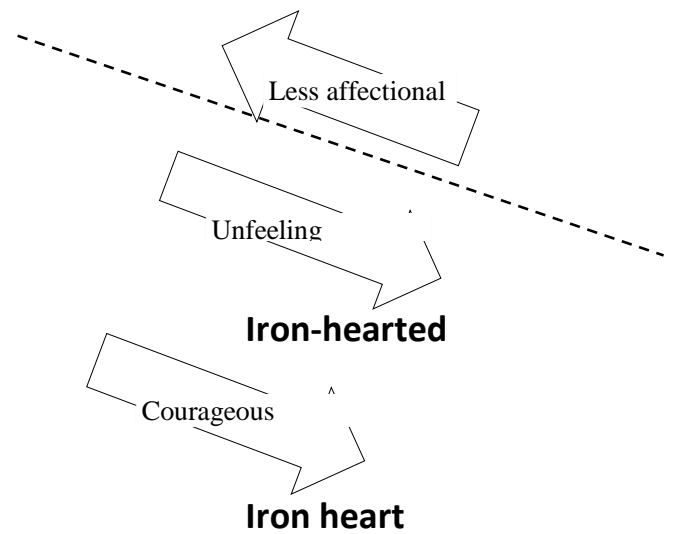

Though both metaphors share similar words, they differ in other values, i.e. the English metaphor acquires the value of "unfeeling" and "cruel" while the Algerian metaphor refers to the "courageous". This difference can be viewed culturally as culture interferes in shaping metaphors towards particular directions. In other words, the lexical interaction is a linguistic mechanism which enables the creation of different words by means of values' interaction as the same word can 
reveal a different meaning when it interacts with a value of another word different from it, and makes communication possible as this emergent meaning becomes rather normative.

When the above metaphors get used in communication, they have the intention of being understood since the mind reading abilities process them easily. However, more semantic ambiguities impose puzzles for their understanding as speakers are not acquainted with, or are shaped in a way often different from the linguistic. More precisely, this includes ambiguous meaning expressed in utterances which hide meaning in a lexical interaction often seen as a semantic density. For this reason, the linguistic analysis of meaning alone is not capable, and a more modular mechanism is needed to approach the hidden intentions, and make meaning of ambiguous utterances more or less clear even if full clarity and real intentions are not possible to confirm and the aim is towards fixing more and more the variability of meaning since the meaning is hidden hides in single as well as multiple ambiguities. To apply the free reasoning, we select to analyze the statement of George W. Bush, "The vast majority of our imports are from outside the country". Let's open it up along the lexical interaction to see what interacts with what. We divide the sentence primarily into main units: [The vast majority] of [our imports] are from [outside] [the country]. These expressions take dictionary definition as follows:

The vast majority

The greater number.

The age at which a person is legally a full adult, usually either 18 or 21 .

The rank or office of a major.

Imports

A commodity, article, or service brought in from abroad for sale.

The implicit meaning or significance of something.

Great significance; importance.

Outside

The outer side, surface, or part; exterior:

The external aspect or appearance.

The Space without or beyond an enclosure, institution, boundary, etc.

\section{Country}

A state or nation

The territory of a nation.

The people of a district, state, or nation:

The land of one's birth or citizenship.

The linguistic analysis cannot find an interaction between the values from their surface level, and as said before, it can just look as nonnormative meaning for the unnecessary repetitions it contains. More pragmatic attributes can be necessary to contextualize the sentence. But with the absence of the pragmatic components this task is difficult, 
and since the meaning of the sentence is put intentionally unclear, the analyst should turn around the sentence from other angles. In that, a search for other "intertexts" is necessary. The same sentence has been said before by George W. Bush, Jr. in Beaverton, Oregon on $25^{\text {th }}$ of September 2000: "More and more of our imports come from overseas"; and by Keppel Enderbery: "Traditionally, most of Australia's imports come from overseas". What has changed in the statement is the word "overseas" which has been replaced by the word "country". And, still, if we rely on the linguistic analysis, "overseas" does not bring anything new. To approach the intended meaning we should first ignore that the statement is a stupidity or just a wrong sentence. The sentence is rather aimed at a much hidden meaning because this president acknowledged that he says that no other man could say. It means he acknowledged ambiguity. Overseas means either a foreign country or can mean over the sea. The US does not import as much as the x-president meant. From the statement, the word "import" is questioned. If it is wrong, then in the mind of the $x$-president another word is meant. "Import" can be turned into a verbal form "what we import". And if "what we import" is replaced by its synonym "what we bring" and the latter turned into the participle form "brought" then "what is brought is from "overseas". Now if "overseas" is a foreign country, the statement becomes "what is brought is from a foreign country". A last decomposition is replacing the word "brought" by "coming". The statement, then, becomes: "the vast majority of what is coming (to the US) is from a foreign country or from an over sea country". Both "a foreign" and "over sea country" refer to a country far from the US and separated by water. It means that the "foreign country" is an attribute of a "strange". And what is strange is not usually a source of goodness but rather the opposite. If this is what the $x$-president meant, then the first part of the expression, in the speaker's mind, is not "the vast majority of our imports" but rather "what comes to the US" which may equal "the strange that comes to the US" are from "a strange country". In other words, the statement summarizes "troubles of the US come from countries foreign to the US".

\section{Approximations}

Ambiguous meaning needs more components than just the syntactic, semantic and the pragmatic because it is the case of a finite mechanism with infinite input. Grice $(1989,23)$ in his "studies in the way of words" described it as "imperfection of natural languages":

For the presence of these elements has the result of both that the concepts within which they appear cannot be precisely or clearly defined and that at least some statements involving them cannot, 
in some circumstances, be assigned definite truth value; and the indefiniteness of these concepts not only is objectionable in itself but also leaves open the way to metaphysics - we cannot be certain that none of these natural language expressions is metaphysically "loaded." [...] the expressions, as used in natural speech, cannot be regarded as finally acceptable, and may turn out to be finally, not fully intelligible.

Grice situates the task of analysis within a metaphysical scope. Semantic indeterminacy, however, joins to metaphysical indeterminacy as the latter advocates to a possible world ${ }^{5}$ absent at the surface level and present in the deep. The search of this world should rely on a mechanism that operates differently from the linguistic, and integrates a free reasoning to operate in a way that decreases ambiguity. The latter lies in the distribution of the attributes around the utterance-word. The distribution is the approximation between the utterance and its meaning. The more the attribute is far the more meaning is ambiguous and the opposite is true. Such a mechanism's main task is to solve the following equation:

$P$ means $Q$

$Q$ is not $P$

$P$ does not mean $Q$. It follows:

It reads $P$ from a surface level.

$P$ is $C M$ (compositional meaning)

Thought to be $Q$.

Q Hides $n(x)$

If it reads $P$ and thought to be $Q$, it means what is hidden is $\mathrm{n}$ and the latter is determined by unknown $\mathrm{x}$ which is to look for along a semantic component in a first stage and a free component in a second. The former, semantic component aims at establishing semantic approximations to set off the point of whether the ambiguous meaning is in the surface structure of the utterance/word or situates in another order. The free component, in contrast, proceeds from a non-linguistic order, and relies on knowledge that the speaker/user of the language thinks appropriate to support a near premise. The latter are raised in order to delimit the search of the right proposition (meaning of the ambiguous utterance/word). If we cast this upon the previously

\footnotetext{
${ }^{5}$ Possible-worlds semantics opens the door for possible meaning that the reader comes to from all possible alternatives including the small and big details that a given world can signify to a given word.
} 
analyzed ambiguities, then the expression "Like beans like lens" is to look at as follows:

It reads $P$ from a surface level

$P$ means that "beans" are like "lens"

$P$ is not $C M$

Thought to be $Q$

$\mathrm{Q}$ hides $\mathrm{n}(\mathrm{x})$

$\mathrm{n}$ is the proposition that:

Beans and lens is $M$

$M$ occurs out of $\mathrm{CM}$

$M$ is a parallel to one of $n_{1}, n_{2}, n_{3}, n_{x}$

$\mathrm{n}_{1}$ answers the question where $\mathrm{M}$ occurs:

If $\mathrm{n}_{1}$ is beans are like lens

$\mathrm{n}_{2}$ isbeans are not like lens

$n_{3}$ is beans are like lens in the context of $x$

$\mathrm{x}$ is the proposition that beans and lens are an exemplification of situations far from the surface meaning.

At this level ends the role of the semantic approximation as it is unable to go along a subjective path. The free component will look for a parallel situation along an established knowledge. With "like beans like lens", the ambiguity is used to avoid saying the right description which is the fact that the political spring is like the regimes before. In fact, this knowledge stems from the socio-cultural development of Arabic as similar comparisons grew out in the form of proverbs: "my lord is like his horse".

The socio-cultural knowledge is less helpful when an utterance is produced in a more closed context, and wherein the parallel situations cannot be reached from elementary semantic approximations. The example of "The vast majority of our imports come from outside the country" is a good case in point. The semantic analysis could say that the intended meaning is the opposite of the compositional meaning, and the variable $\mathrm{n}$ is the proposition "strange" since the word "import" has "strange" as one of its far attributes. The free component in Bush's sentence is the choice we made in selecting the variable n. "Strange" is selected because previous antecedents favour this proposition. They are: "More and more of our imports come from overseas" and "Traditionally, most of Australia's imports come from overseas". They are, in fact, historical intertexts which could delimit the semantic approximation by putting emphasis on the word "import". The latter brought further approximations which are led to the final meaning "a strange country". 
What has been said so far on semantic approximation relies, in fact, on a first stage which is the utterance/word itself. Despite its ambiguous meaning at the surface structure, the navigation along the semantic and free component could still proceed along delimiting the propositions. At the outset, the free reasoning takes the following paths:

1. It looks for possible relations between words including the linguistic and non-linguistic knowledge;

2. It is open to atypical attribution of meaning; and

3. It goes beyond the semantic and pragmatic regularities.

Let's take the following sentence said by Pablo Picasso and considered as an ambiguity, to see how to work out its meaning by relying on substitution of attributes and approximations.

(6) Everything you can imagine is real ${ }^{6}$.

The sentence in its first reading states the idea that all what we think about is real, or what is in the mind exists out of the mind. As such, the sentence is semantically unaccepted because of abstract words. However, there are three words that turned the sentence meaning and hide the real meaning. They are "everything", "real" and "imagine". Why not "can" and "imagine"? Simply because "can" should be here to cover the mind's capacity of imagination.

And "imagine" is a word that is the second component of the equation: $x_{1}=x_{2}$

Everything you can imagine $=$ real

"Everything" in the sentence is a substitution of the expressions "anything" and "all things". That is, anything the mind may draw pictures of. It is the interpretation of $-\infty \infty+$. And this is true as the mind's capacity covers the very smallest and the very biggest things in the world, and fictional science proved that so. Now, the word "imagine" is a substation of the words "create" and "realize"; it loses its primary meaning of thinking and conceptualizing and takes instead a practical meaning: create and realize. A question here should be? why this choice? The answer lies now on approximations which are first in the field of the sentence, that is its semantic field which is painting. Painting is the creation and production results from imagination and conceptualization. However, this is not enough as another approximation is needed to fully confirm the worked out meaning. It lies in the word "real". The latter has two attributes: "The act of realizing" and "the act of making real". Both work together. The first attributes are compatible with the worked out meaning. First, we take "The act of realizing" which leads to the approximation with "the act of creating and

${ }^{6}$ Pablo Picasso, Spanish Cubist painter (1881 - 1973) 
realizing": "all what you imagine is real" reads as "all what you can create is real". In other words, the sentence meaning is you can create anything and turn it real. If we cast this upon the field of art in general, which is the semantic field of the sentence, the worked out meaning resulting from substitution of attributes and approximation is: what you can think about is what you can create and it is as original as the real world, that is, making the unreal (imaginary) real.

Another example from poetics is supposed to clarify more the task of the free reasoning since literature is known for intentional ambiguity whereby the literary genre has semantic ambiguity frequent to enrich meaning in the way suggested by the semantic theory (Olsen13). In literature, ambiguous meaning is neither true nor false till a given literary context justifies it. Ambiguity may occur in the text without being itself ambiguous because its indeterminacy lies in the inability of the reader to understand its presence and function. The following extract from William Faulkner's The Sound and the Fury contains an ambiguity that has been recognized as "unreached meaning":

Through the fence, between the curling flower spaces, I could see them hitting. They were coming towards where the flag was and I went along the fence. Luster was hunting in the grass by the flower tree. They took the flag out, and they were hitting. They put the flag back and they went to the table, and he hit and the other hit. $(I, 11)$

All seems ordinary except the sentences "They took the flag out, and they were hunting" and "They put the flag back and they went on". The latter are irrelevant to the extract because it is not known why and how "hunting" and "fly" relate to the children's playing in the fence. Literary critics explained the sentence as a nonrealization of the direct object "hit" as the result of the narrator's madness -Benjy - which implies the lost action (Wilson 29). The ambiguity has been also seen as a mind style defined by Fowler (1977) as: "Cumulatively, consistent structural options, agreeing in cutting the presented world to one pattern or another, give rise to an impression of a world view, what I shall call a 'mind style"' (Semino and Culpeper-96). The other world that justifies Benjy's utterance is literarily a mind style the author used as Benjy's tongue. Saying this, however, is situating the utterance in its area which is incapable of decoding the writer's meaning since the utterance "putting in and down the flag" remains ambiguous. Such utterances seem to have "static attributes" holding a meaning known only by the writer and can hardly be discerned by the reader. The semantic analysis does not stand only in inscribing the ambiguous 
utterance to a literary context. It needs rather to provide meaning. Thus, literary language escapes to the systemic approach and needs another modeling. Looking at the above "indeterminacy" from the present suggested model may contribute to decreasing the indeterminacy because it operates from a free scheme, and, at the same time interactional in the sense that any item that attributes an interaction from near or far with one of the items of the indeterminacy is signaled.

The main attributive words in "They took the flag out, and they were hitting. They put the flag back and they went to the table, and he hit and the other hit" are "flag", "hit", "out", "back". But since the utterance's meaning will start from a zero state due to its full indeterminacy, other functional words need to enter in the interaction of attributes because they can be semantic attributes. They are "he" and "they". In the first sentences "They took the flag out, and they were hitting", there is no link between "They took the flag out" and "they were hitting" especially because the verb "hit" lacks its object. The second sentence "They put the flag back and they went to the table" seems similar to the first sentence in putting and taking out the flag and putting it back after an "action" which is said in the first sentence "hitting" and in the second as "went to the table". "They hit when the flag is out and they do not when it is on". This implies that putting the flag out is permission for hitting. But right now the utterance's meaning is still vague as the question remains "hitting what?". From the utterance itself, no clear meaning can yield. The words in the target utterance do not relate to each other in a particular interaction and what could be obtained is just a correlation between the flag and hitting. To proceed in a further analysis of the attributes of meaning, other extensions should be applied by looking for intertexts from near or far. At this level, the analysis is beyond the reader-author cooperation as the linguistic material can no longer act as a source of meaning. The context, too, can add nothing because it is itself unrelated to the utterance's meaning.

The red or yellow flag is generally put in the beach to indicate that swimming is forbidden. And in the ambiguous sentence the flag is out when they hit. A parallel between the two utterances implies that when they hit, the flag is out and when they do not hit the flag is back. The flag can refer to the permission of hitting. The question of "what did they hit" is a puzzle. After returning to the table, hitting is still recurrent: "and he hit and the other hit". Two possibilities arise here: what they hit near to the flag is it the same found in the table? Or it is different? The second possibility is more probable because the co-text "flag out, flag back" 
conditions hitting. But if we carry on reading the passage, it seems that "hitting" continues in different other places:

They were hitting little, across the pasture. I went back along the fence to where the flag was. It flapped on the bright grass and the trees (1).

On page 48, the verb "hit" figures out with relation to the flag:

They came to the flag. He took it out and they hit, then he put the flag back.

"Mister." Luster said.

He looked around. "What." he said.

"Want to buy a golf ball." Luster said.

"Let's see it." he said. He came to the fence and Luster reached the ball through.

"Where'd you get it." he said.

"Found it." Luster said.

"I know that." he said. "Where. In somebody's golf bag." (3)

The first sentence "They came to the flag. He took it out and they hit, then he put the flag back" reoccur in the target utterance. In the rest, "hit" refers to a ball. So, is the ball the object of "hit"? The answer is no because Luster talked about the ball as something different from what "hit" refers to in the first sentence. At this level, we can confirm that the ambiguous utterances "They took the flag out, and they were hitting. They put the flag back and they went to the table, and he hit and the other hit" are said independently of the co-text and their words are put superficially with signifiers that have no link with the words. The sentence is altogether referring to a meaning of an action performed in condition to another action. The sentence is a nonrealization as literary critics view it. The interactional mechanism which tried to collect all possible attributes could say that the sentence's meaning is a realization of another sentence that the writer does not communicate. Faulkner put it ambiguous on purpose: "But when I wrote Benjy's section, I was not writing it to be printed". It means not to be read. In other words, the linguistico-pragmatic cooperation could surround the ambiguity from different angles of which is the fact that all the words in the sentence lack relationships with their signified or are simply asignified because indeterminacy, here, is brought to a highest degree, and the words of the sentence have nothing to do with the text. 


\section{Conclusion}

Ambiguity is a semantic indecision of utterances intended by language users. It varies in the degree of indeterminacy. It has been approached by the linguistic analysis but the latter has been shown to be unable to cover the variability of the linguistic input given that the producer of the indeterminate meaning and its receiver lack the same coding, and the latter is not conventional. What is needed for such meaning is a mechanism that is both dynamic in the collection of attributes and interactional in the sense that it establishes possible interactions towards clarifying indeterminacy.

The present paper suggested an extensional model that stands principally on delimiting the openness of ambiguity by integrating an open free reasoning which is likely to proceed from a logical reasoning yet not necessarily linguistic. The role of this reasoning acts freely from the restricted linguistic rules which fail to turn around the utterance from several angles that shape meaning. The free reasoning is a component added to the semantic-pragmatic analysis for the aim of finding attributes of meaning from unpredictable ways which can employ a logical reasoning able to yield meaning.

\section{Works Cited:}

Blommaert, Jan. Discourse: A Critical Introduction. Cambridge UP, 2005.

Carston, Robyn. "The semantics/pragmatics distinction: a view from relevance theory". Working Papers in Linguistics 10 (1998): 1-30.

Cook, Guy. "Use of translation in language teaching."Routledge Encyclopedia of Translation Studies. Ed. Mona Baker. New York and London: Routledge, 2001. 117-120.

Cruse, David Alan. Lexical Semantics. Cambridge UP, 1986.

Dayal, Veneeta. "The Universal Force of Free Choice Any". Linguistic Variation Yearbook 4 (2004): 5-40.

Derrida. Jaques. L'écriture et la Différence. Éditions du Seuil, 1967.

Faulkner, William. The Sound and the Fury.Penguin Modern Classics, 1964.

Gibbs, Raymond. W."The emergence of intentional meaning: a different twist on pragmatic linguistic action". Lodz Papers in Pragmatics 8.1 (2012):1735.

Grice, Paul. Studies in the Way of Words. Harvard UP, 1989.

Katz, Jerrold. J."Semantic theory."Semantics: An Interdisciplinary Reader in Philosophy, Linguistics and Psychology. Ed. Danny David Steinberg. CUP Archive, 1971. 297-307.

Olsen, Stein. Haugom. The Structure of Literary Understanding. CUP Archive, 1985.

Recanati, François. Literal Meaning. Cambridge UP, 2004.

Semino, Elena and Culpeper, Jonathan. Cognitive Stylistics: Language and cognition in text analysis. John Benjamins Publishing Campany, 2002. 
Stampe, Dennis. "Pragmatics and causal theoretic aspects of semiotics."Possibilities and limitations of pragmatics. Eds. Herman. Parret. Marina. Sbisa and Jef. Verschueren. John Benjamins Publishing Campany, 1981. 683-712.

Szab'o, Zolt' an Gendler. The distinction between semantics and pragmatics. https://webcache.googleusercontent.com/search?q=cache:cveTnte54b8 $\mathrm{J}:$ https://philpapers.org/rec/SZASVP+\&cd=2\&hl=fr\&ct=clnk\&gl=tn $\quad[20-$ 06-2019]. 2006.

Szab' o, Zolt' an Gendler. Problems of Compositionality. Routledge, 2014.

Toolan, Michael. Narrative progression in the short story: a corpus stylistic approach. John Benjamins Publishing Company, 2009.

Van Orden, Guy.C and Holden, John.G. "Intentional Contents and Self-Control". Ecological Psychology 14(1-2) (2002): 87-109.

Wales, K. "Ambiguity". Concise Encyclopedia of Philosophy of Language. Ed. P. Lamarque. Elsevier Science, 1997. 387.

Wilson, John. Talking with the President: The Pragmatics of Presidential Language. Oxford UP, 2015.

Wilson, Peter. Mind the Gap: Ellipsis and Stylistic Variation in Spoken and Written English. Routledge, 2014.

Wilson, Deirdre and Sperber, Dan. Meaning and Relevance. Cambridge UP, 2012.

\section{PREISPITIVANJE DVOSMISLENOSTI IZVAN VEZE SEMANTIKE I PRAGMATIKE}

Dvosmislenost nastaje kao posljedica intencije govornika da se izrazi nejasno ili nedovoljno konkretno. Pristupili smo joj sa stanovišta semantike, pragmatike i filozofije jezika, jer neodređenost u značenju zahtijeva mehanizam koji će grupisanjem odgovarajućih atributa dovesti do njenog prevazilaženja. U ovom radu ukazali smo na mehanizam interekcije koji integriše dvije komponente. Prva se oslanja na lingvističke izvore moguće atribucije, a druga je komponenta slobodna, pa analitičar za proširenje atributa i njihovu interakciju ne mora nužno da slijedi lingvistička pravila. Ovaj je mehanizam primjenjen na dvosmislenim iskazima širokog spektra - od najjednostavnijih dnevnih metafora do proizvoljnih iskaza. Utvrđeno je da je je mehanizam primjenjiviji što je atribut značenjski udaljeniji.

Ključne riječi: dvosmislenost, lingvistička analiza, interakcija, pragmatika, semantika, substitucija, aproksimacija 